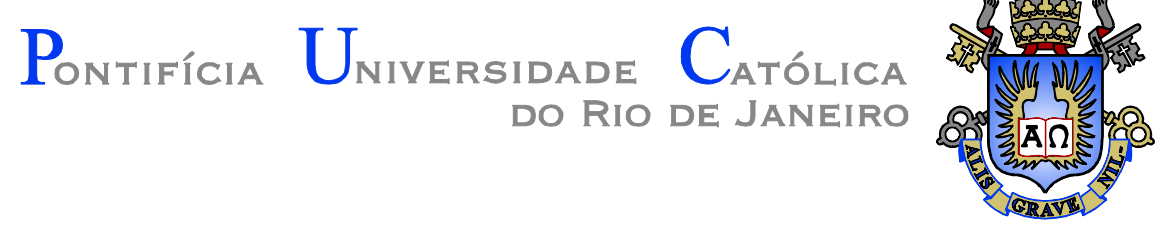

Edwin Ronald Valderrama Campos

Modelagem do Uso de Nanofluidos no Sistema de Arrefecimento de Motores a Combustão Interna

Dissertação de Mestrado

Dissertação apresentada como requisito parcial para obtenção do título de Mestre pelo Programa de PósGraduação em Engenharia Mecânica da PUC-Rio.

Orientador: Prof. José Alberto dos Reis Parise Co-Orientador: Dr. Frank Chaviano Pruzaesky

Rio de Janeiro

Outubro de 2009 


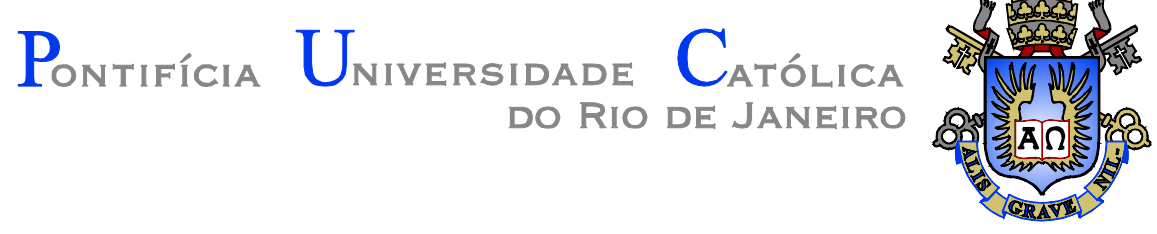

Edwin Ronald Valderrama Campos

\section{Modelagem do Uso de Nanofluidos no Sistema de Arrefecimento de Motores a Combustão Interna}

Dissertação apresentada como requisito parcial para obtenção do título de Mestre pelo Programa de Pós-Graduação em Engenharia Mecânica da PUC-Rio. Aprovada pela Comissão Examinadora abaixo assinada.

Prof. José Alberto dos Reis Parise Orientador

Departamento de Engenharia Mecânica - PUC-Rio

Prof. Frank Chaviano Pruzaesky Co-Orientador Departamento de Engenharia Mecânica - PUC-Rio

Prof. Sergio Leal Braga Departamento de Engenharia Mecânica - PUC-Rio

Prof. Carlos Valois Maciel Braga Departamento de Engenharia Mecânica - PUC-Rio

Prof. Carlos Eduardo Leme Nóbrega CEFET/RJ

Prof. José Eugenio Leal Coordenador Setorial do Centro Técnico Científico - PUC-Rio

Rio de Janeiro, 30 de outubro de 2009 
Todos os direitos reservados. É proibida a reprodução total ou parcial do trabalho sem autorização da universidade, do autor e do orientador.

\section{Edwin Ronald Valderrama Campos}

Graduou-se em Engenharia Mecânica no Dpto. De Engenharia Mecânica da UNT (Universidad Nacional de Trujillo, Trujillo - Perú) em 2006.

Ficha Catalográfica

Campos, Edwin Ronald Valderrama

Modelagem do uso de nanofluidos no sistema de arrefecimento de motores a combustão interna / Edwin Ronald Valderrama Campos; orientadores: José Alberto dos Reis Parise, Frank Chaviano Pruzaesky. - 2009.

97 f.: il. (color.) ; $30 \mathrm{~cm}$

Dissertação (Mestrado em Engenharia Mecânica)Pontifícia Universidade Católica do Rio de Janeiro, Rio de Janeiro, 2009.

Inclui bibliografia.

1. Engenharia mecânica - Teses. 2. Nanofluidos. 3. Motor de combustão interna. 4. Fluido de arrefecimento. 5. Radiador automotivo. 6. Arrefecimento de motor. 7. Bomba de líquido. I. Parise, José Alberto dos Reis. II. Pruzaesky, Frank Chaviano. III. Pontifícia Universidade Católica do Rio de Janeiro. Departamento de Engenharia Mecânica. IV. Título.

CDD: 621 
Aos meus pais, e aos meus amigos Jose e Sara. 


\section{Agradecimentos}

A Deus por cada novo dia.

Aos meus orientadores, Professor José Alberto dos Reis Parise, e Doutor Frank Chaviano Pruzaesky, por sua dedicação, confiança e amizade concedida ao longo do curso.

Ao Professor Sergio Libanio do CEFET-RJ pelo seu apoio durante a realização do presente trabalho.

Ao colega Juan Carlos Valdez Loaiza pela ajuda na elaboração do documento final.

Aos professores do Departamento de Engenharia Mecânica da PUC-Rio pelos ensinamentos que contribuíram à minha formação.

Aos colegas e funcionários pela hospitalidade e apoio.

A todos os amigos que, com seu apoio e amizade, contribuíram para que minha permanência no Brasil seja muito agradável.

Finalmente, minha gratidão ao CNPq e à PUC-Rio pelos auxílios concedidos, sem os quais este trabalho não poderia ter sido realizado. 


\section{Resumo}

Campos, Edwin Ronald Valderrama; Parise, José Alberto dos Reis; Pruzaesky, Frank Chaviano. Modelagem do Uso de Nanofluidos no Sistema de Arrefecimento de Motores a Combustão Interna. Rio de Janeiro, 2009. 97p. Dissertação de Mestrado - Departamento de Engenharia Mecânica, Pontifícia Universidade Católica do Rio de Janeiro.

Estudou-se a aplicação de nanofluidos no sistema de arrefecimento de motores a combustão interna. Nanofluidos são suspensões de partículas de diâmetro menor que $100 \mathrm{~nm}$ em fluidos convencionais de troca de calor, tais como água, óleo, etileno glicol, entre outros. Devido às suas características favoráveis de transferência de calor, em função da suspensão de partículas, metálicas ou não metálicas, com elevada condutividade térmica, nanofluidos têm sido considerados para atuar como fluidos térmicos em diferentes aplicações. Desenvolveram-se modelos matemáticos para operação em regime permanente, na avaliação do efeito das características térmicas e hidráulicas do escoamento do nanofluido nos componentes do sistema de arrefecimento; e em regime transiente, na avaliação do processo de aquecimento do motor. Fez-se uso do pacote EES para a simulação e consideraram-se os seguintes componentes do sistema de arrefecimento automotivo: radiador, camisas do bloco de cilindros, termostato e bomba do líquido de arrefecimento. Foram empregados o método dos parâmetros concentrados e o método $\varepsilon-\mathrm{NTU}$ para a modelagem global do sistema monofásico. Diferentes tipos de nanofluidos, com variações na concentração volumétrica de nanopartículas, foram considerados na avaliação desta alternativa em fluidos térmicos visando aplicações automotivas.

\section{Palavras-chave}

Nanofluidos; Motor de Combustão Interna; Fluido de Arrefecimento; Radiador Automotivo; Arrefecimento do Motor; Bomba de Líquido. 


\section{Abstract}

Campos, Edwin Ronald Valderrama; Parise, José Alberto dos Reis (Advisor); Pruzaesky, Frank Chaviano. Modeling of the use of nanofluids in internal combustion engines cooling systems. Rio de Janeiro, 2009. 97p. MSc Dissertation - Departamento de Engenharia Mecânica, Pontifícia Universidade Católica do Rio de Janeiro.

The application of nanofluids in cooling systems of internal combustion engines was studied. Nanofluids consist of nanoparticles (with dimension below $100 \mu \mathrm{m}$ ) suspended in traditional heat transfer fluids, such as water or ethylene glycol. Given their favourable heat transfer characteristics, because of the suspension of high thermal conductivity particles, metallic or non-metallic, nanofluids have been considered as potential substitutes for conventional heat transfer fluids. Mathematical models were developed for steady-state operation, for the evaluation of thermal and hydraulic behavior of the cooling system, and for transient regime, for the assessment of the engine start-up process. The EES software was employed for the simulation. The following components of the cooling system were considered: radiator, engine cooling jackets, thermostat and coolant pump. Lumped parameter analysis and the effectivenessNTU method were used for the single-phase system simulation. Different types of nanofluids, with variation on the volume fraction, were considered in this study.

\section{Keywords}

Nanofluids; Internal Combustion Engine; Coolant; Automotive Radiator; Engine Cooling; Coolant Pump. 


\section{Sumário}

Lista de Símbolos

1 Introdução

1.1. Objetivo

1.2. Justificativa

1.3. Contribuição do presente trabalho

1.4. Conteúdo do trabalho

2 Uma breve revisão sobre nanofluidos

2.1. Produção de nanopartículas e nanofluidos 23

2.2. Propriedades dos nanofluidos $\quad 27$

2.2.1. Condutividade térmica efetiva 27

2.2.2. Viscosidade dinâmica 30

2.2.3. Densidade e calor específico 32

3 Estado da arte do sistema de arrefecimento automotivo 33

3.1. Componentes e funcionamento de um sistema de arrefecimento automotivo

3.2. O calor no sistema de arrefecimento automotivo 35

3.2.1. Balanço de energia

3.2.2. Mecanismos de transmissão de calor no motor a combustão interna

3.3. A camisa de água do motor

3.4. O radiador automotivo

3.5. A bomba do fluido de arrefecimento

3.6. O termostato

3.7. Fluidos de arrefecimento

3.8. Nanofluidos em sistemas de arrefecimento automotivos

4 Modelo matemático do sistema de arrefecimento de um motor a 
combustão interna $\quad 49$

4.1. Descrição do sistema proposto 49

4.2. Equações de conservação 50

4.2.1. Jaqueta do motor 51

4.2.2. Radiador 52

4.2.3. Bomba do fluido de arrefecimento 53

4.3. Modelo matemático das jaquetas do motor 53

4.4. Modelo matemático do radiador 54

4.4.1. Taxa de transferência de calor 55

4.4.2. Coeficiente convectivo de troca de calor do ar 57

4.4.3. Coeficiente convectivo de troca de calor do fluido de resfriamento $\quad 59$

4.4.4. Queda de pressão no lado do ar 62

4.4.5. Queda de pressão no lado do fluido de resfriamento 62

4.4.6. Potência de bombeamento do fluido de resfriamento 63

5 Modelo do regime transiente durante o aquecimento de um motor a combustão interna 64

5.1. Descrição do sistema para o regime transiente 64

5.2. Desenvolvimento do modelo do regime transiente 66

5.2.1. Sistema: fluido de arrefecimento 66

5.2.2. Sistema: bloco do motor 67

6 Métodos de solução $\quad 69$

6.1. Modelo para regime permanente 69

6.1.1. Dados de entrada 69

6.1.2. Dados de saída $\quad 71$

6.1.3. Plataforma computacional 72

6.1.4. Desenvolvimento do código 73

6.1.5. Estrutura do programa $\quad 74$

6.2. Solução numérica do modelo do regime transiente 75

7 Resultados do modelo do sistema de arrefecimento do motor de combustão interna 
$\begin{array}{ll}\text { 7.1. Propriedades termofísicas } & 79\end{array}$

$\begin{array}{ll}\text { 7.1.1. Densidade efetiva } & 79\end{array}$

7.1.2. Calor específico efetivo 79

7.1.3. Viscosidade dinâmica 80

7.1.4. Condutividade térmica efetiva 82

7.2. Resultados para o radiador 82

7.2.1. Número de Nusselt do nanofluido 83

7.2.2. Coeficiente de troca de calor 83

7.2.3. Área requerida de transferência de calor 85

7.2.4. Altura do radiador 86

7.3. Bomba do fluido de arrefecimento 86

7.3.1. Queda de pressão 17

7.3.2. Potência de bombeamento $\quad 87$

7.4. Resultados do modelo do regime transiente 89

8 Conclusões e recomendações 91

$\begin{array}{ll}\text { Referências bibliográficas } & 93\end{array}$ 


\section{Lista de figuras}

Figura 1 - Condutividade térmica de algumas substâncias com respeito à condutividade térmica da água, baseado em Wang e Mujumdar (2008).

Figura 2 - Sistema de produção de nanopartículas pelo método de fase gasosa incrementado por plasma (Hosokawa et al., 2007).

Figura 3 - Nanopartículas de $79 \% \mathrm{Al}_{2} \mathrm{O}_{3}-21 \% \mathrm{SiO}_{2}$

(Hosokawa et al., 2007).

25

Figura 4 - Esquema da produção de nanofluidos por evaporação condensação de vapor metálico (Choi e Eastman, 2001).

Figura 5 - Esquema do homogenizador de alta pressão para a produção de nanofluidos em dois passos (Hwang et al., 2008).

Figura 6 - Vista secionada de um motor V-8, mostrando o sistema de arrefecimento. As setas brancas indicam o fluxo do FDA através da camisa do motor (Crouse e Anglin, 1977).

Figura 7 - Diagrama do fluxo de energia para um motor a combustão interna (Heywood, 1988).

Figura 8 - (a) Distribuição de temperaturas medidas (pontos) e calculadas no pino e no lado de carga do pistão e; (b) Porcentagem do fluxo de calor em diferentes zonas do pistão (Heywood, 1988).

Figura 9 - Vista secionada do bloco de cilindros de um motor V-6 mostrando as camisas do motor (Crouse e Anglin, 1977).

Figura 10 - Fluxo de calor através das paredes da câmara de combustão (Heywood, 1988).

Figura 11 - Geometrias típicas de radiadores automotivos.

Figura 12 - Seção dos canais inclinados das aletas mostrando os dois tipos de fluxos do ar, através do duto e através dos canais inclinados (Cowell et al., 1995).

Figura 13 - Bomba do fluido de arrefecimento: (a) marca Daihatsu, 2009 e; (b) vista em seção mostrando o eixo e o rolamento de esferas de fileiras duplas (Crouse e Anglin, 1977). 
Figura 14 - Tipos de termostato.

Figura 15 - Estrutura do trocador de calor compacto estudado por Vasu et at. (2008).

Figura 16 - Esquema simplificado do sistema de arrefecimento do motor de combustão interna.

Figura 17 - Esquema do radiador e o sentido dos fluxos, cruzados e não misturados, envolvidos no processo de troca de calor.

Figura 18 - Esquema do circuito usado para o modelo do regime transiente, mostrando o sistema ativado durante o aquecimento do motor (linha cheia), e o circuito utilizado durante a operação normal do motor (linha tracejada).

Figura 19 - Interpretação gráfica do método de Newton - Raphson (Kelley, 2003).

Figura 20 - Esquema do processo de cálculo pelo método de Euler.

Figura 21 - Densidade efetiva dos nanofluidos para várias concentrações de nanopartículas com diâmetro $d_{n p}=10 \mathrm{~nm}$ operando a $89{ }^{\circ} \mathrm{C}$.

Figura 22 - Calor específico efetivo dos nanofluidos para diferentes frações volumétricas de nanopartículas com diâmetro $d_{n p}=10 \mathrm{~nm}$ e operando a $89^{\circ} \mathrm{C}$.

Figura 23 - Viscosidade dinâmica efetiva para várias concentrações de nanopartículas com um diâmetro $d_{n p}=10 \mathrm{~nm}$ e operando a $89^{\circ} \mathrm{C}$. Figura 24 - Condutividade térmica efetiva para diferentes frações volumétricas de nanopartículas, com um diâmetro $d_{n p}=10 \mathrm{~nm}$ e operando a $89^{\circ} \mathrm{C}$.

Figura 25 - Número de Nusselt do nanofluido escoando nos tubos de seção alongada em função da concentração volumétrica de nanopartículas com diâmetro $d_{n p}=10 \mathrm{~nm}$ e operando a $89{ }^{\circ} \mathrm{C}$.

Figura 26 - Coeficiente de troca de calor para diferentes concentrações de nanopartículas, a uma temperatura de operação de $89^{\circ} \mathrm{C}$, e um diâmetro de nanopartículas $d_{n p}=10 \mathrm{~nm}$.

Figura 27 - Área de transferência de calor no lado interno dos tubos de seção oval do radiador para diferentes concentrações, a uma 
temperatura de operação de $89{ }^{\circ} \mathrm{C}$, e para um diâmetro de partículas $d_{n p}=10 \mathrm{~nm}$.

Figura 28 - Altura do radiador para diferentes concentrações de nanopartículas, para uma temperatura de operação de $89{ }^{\circ} \mathrm{C}$ e um diâmetro de partículas $d_{n p}=10 \mathrm{~nm}$.

Figura 29 - Queda de pressão dos nanofluidos em função da concentração volumétrica das nanopartículas, a uma temperatura de operação de $89{ }^{\circ} \mathrm{C}$ e um diâmetro de partículas $d_{n p}=10 \mathrm{~nm}$.

Figura 30 - Potência de bombeamento do nanofluido a diferentes concentrações volumétricas, a uma temperatura de operação de $89{ }^{\circ} \mathrm{C}$, e um diâmetro de nanopartículas $d_{n p}=10 \mathrm{~nm}$.

Figura 31 - Efeito da concentração das nanopartículas sobre a potência de bombeamento para nanofluido de alumina (Ben Mansour et al., 2007).

Figura 32 - Aumento da temperatura do FDA durante o período de aquecimento do motor (termostato fechado) para o nanofluido $\mathrm{H}_{2} \mathrm{O}$ - Cu e água.

Figura 33 - Aumento da temperatura do FDA durante o período de aquecimento do motor (termostato fechado) para o nanofluido $\mathrm{H}_{2} \mathrm{O}-\mathrm{Al}_{2} \mathrm{O}_{3}$ e água. 


\section{Lista de tabelas}

Tabela 1 - Valor da constante $c$ da eq. (2.7) para diferentes nanofluidos (Velagapudi, V. et al., 2008). 30

Tabela 2 - Propriedades termofísicas das nanopartículas,

(Velagapudi, V. et al., 2008). 


\section{Lista de Símbolos}

\section{Símbolos gerais}

\begin{tabular}{|c|c|}
\hline$A$ & Área, $\left[\mathrm{m}^{2}\right]$ \\
\hline$A_{c}$ & Área de fluxo-livre mínima no lado do ar do radiador, $\left[\mathrm{m}^{2}\right]$ \\
\hline$A_{\text {fr }}$ & Área frontal do radiador, $\left[\mathrm{m}^{2}\right]$ \\
\hline$A_{r}$ & Razão de aspecto dos tubos de seção alongada $\left(A_{r}=T_{w} / T_{d}\right)$, [-] \\
\hline$A_{t b}$ & Área da seção transversal dos tubos de seção alongada, $\left[\mathrm{m}^{2}\right]$ \\
\hline$A_{w}$ & Área média de troca de calor nos tubos de seção alongada, $\left[\mathrm{m}^{2}\right]$ \\
\hline$b$ & Comprimento médio da distância entre aletas, [m] \\
\hline C & Taxa de capacidade térmica $\left(\dot{m} c_{p}\right),[\mathrm{kW} / \mathrm{K}]$ \\
\hline$c_{p}$ & Calor específico a pressão constante, $[\mathrm{kJ} / \mathrm{kg} \cdot \mathrm{K}]$ \\
\hline$C_{r}$ & Razão das taxas de capacidade térmicas $\left(C_{r}=C_{\min } / C_{\max }\right),[-]$ \\
\hline$D_{h}$ & Diâmetro hidráulico, [m] \\
\hline$D_{p s t}$ & Diâmetro do pistão, [m] \\
\hline$D_{\text {rd }}$ & Espessura do radiador, [m] \\
\hline$D_{t b}$ & Diâmetro das tubulações, [m] \\
\hline$f$ & Fator de atrito de Fanning, [-] \\
\hline$F$ & Razão de combustível - ar, [-] \\
\hline$F_{d}$ & Comprimento das aletas no sentido do fluxo do ar, [mm] \\
\hline$F_{e}$ & Eficiência de fluxo, [-] \\
\hline$F_{l}$ & Comprimento das aletas, $[\mathrm{mm}]$ \\
\hline$F_{p}$ & Passo das aletas, $[\mathrm{mm}]$ \\
\hline$F_{t h}$ & Espessura das aletas, [mm] \\
\hline$G$ & Velocidade mássica, $\left[\mathrm{kg} / \mathrm{m}^{2}-\mathrm{s}\right]$ \\
\hline$H_{r d}$ & Altura do radiador, [m] \\
\hline j & Fator de Colburn $\left(j=\alpha / G c_{p}\right),[-]$ \\
\hline k & Condutividade térmica, $[\mathrm{kW} / \mathrm{m} \cdot \mathrm{K}]$ \\
\hline$L_{1}$ & Comprimento dos louvers, [m] \\
\hline$L_{p}$ & Passo dos louvers, [m] \\
\hline
\end{tabular}




$\begin{array}{ll}L_{r d} & \text { Comprimento do radiador, [m] } \\ L_{t b} & \text { Comprimento das tubulações, [m] } \\ m & \text { Parâmetro da eficiência da aleta, }\left[\mathrm{m}^{-1}\right] \\ \dot{m} & \text { Vazão mássica, [kg/s] } \\ N_{t b} & \text { Número de tubos no radiador, [-] } \\ N T U & \text { Unidades de transferência de calor, [-] } \\ N u & \text { Número de Nusselt, [-] } \\ P & \text { Pressão, [kPa] } \\ P r & \text { Número de Prandlt, [-] } \\ \dot{Q} & \text { Calor fornecido ao sistema de arrefecimento, [kW] } \\ R e & \text { Número de Reynolds baseado no diâmetro hidráulico, [-] } \\ S_{p s t} & \text { Curso do pistão, [m] } \\ S_{1} & \text { Comprimento dos louver de entrada e saída, [m] } \\ S_{2} & \text { Comprimento do louver defletor, [m] } \\ T & \left.\text { Temperatura, [ }{ }^{\circ} \mathrm{C}\right] \\ T_{d} & \text { Comprimento dos tubos no sentido do fluxo do ar, [m] } \\ T_{f} & \left.\text { Temperatura fria do fluido de arrefecimento, [ }{ }^{\circ} \mathrm{C}\right] \\ T_{g} & \left.\text { Temperatura média efetiva do gás, [ }{ }^{\circ} \mathrm{C}\right] \\ T_{p} & \text { Passo dos tubos de seção alongada, [m] } \\ T_{q} & \left.\text { Temperatura quente do fluido de arrefecimento, [ }{ }^{\circ} \mathrm{C}\right] \\ T_{t h} & \text { Espessura da parede dos tubos de seção alongada, [m] } \\ T_{w} & \text { Comprimento dos tubos de seção alongada, [m] } \\ U & \left.\text { Coeficiente geral de transferência de calor, [kW/m }{ }^{2} \cdot \mathrm{K}\right] \\ U_{c} & \text { Velocidade na área de fluxo-livre mínima, [m/s] } \\ u_{t r} & \text { Velocidade frontal do ar, [m/s] } \\ U_{n f} & \text { Velocidade do nanofluido, [m/s] } \\ \dot{W} & \text { Potência de bombeamento, [kW] } \\ & \end{array}$

\section{Símbolos gregos}

a Coeficiente de troca de calor, $\left[\mathrm{kW} / \mathrm{m}^{2} \cdot \mathrm{K}\right]$

$\beta$

Razão entre a área total de troca de calor de um lado só e o volume total do radiador, $\left[\mathrm{m}^{2} / \mathrm{m}^{3}\right]$ 


$\begin{array}{ll}\gamma & \text { Razão entre a área aletada de um lado só e a correspondente } \\ \Delta & \text { área total de troca de calor, [-] } \\ \varepsilon & \text { Diferença } \\ \eta & \text { Efetividade, [-] } \\ \mu & \text { Eficiência, [-] } \\ \rho & \text { Viscosidade dinâmica, }[\mathrm{Pa} \cdot \mathrm{s}] \\ \sigma & \text { Densidade, }\left[\mathrm{kg} / \mathrm{m}^{3}\right] \\ v & \text { Razão entre a área de fluxo-livre mínima e a área frontal do } \\ \zeta & \text { radiador }\left(\sigma=A_{c} / A_{f r}\right),[-] \\ & \text { Viscosidade cinemática }(v=\mu / \rho),\left[\mathrm{m}^{2} / \mathrm{s}\right] \\ & \text { Difusividade térmica }\left(\zeta=k / \rho c_{p}\right),\left[\mathrm{m}^{2} / \mathrm{s}\right]\end{array}$

\section{Símbolos subscritos}

$\begin{array}{ll}\text { ar } & \text { Geral } \\ b c & \text { Bloco do motor } \\ f & \text { Aleta } \\ f b & \text { Fluido base } \\ f d & \text { Completamente desenvolvido } \\ i & \text { Ingresso } \\ l a m & \text { Regime laminar } \\ m & \text { Média } \\ \text { max } & \text { Máxima } \\ \text { min } & \text { Mínima } \\ n f & \text { Fluido de arrefecimento (nanofluido) } \\ 0 & \text { Saída } \\ r d & \text { Radiador } \\ \text { turb } & \text { Regime turbulento } \\ \text { wall } & \text { Parede dos tubos de seção alongada no radiador }\end{array}$


"Ciência é duvidar da autoridade dos especialistas" Richard Feynman (Nobel de Física, 1965) 